\title{
BMJ Open Experiences and supportive care needs of UK patients with pancreatic cancer: a cross-sectional questionnaire survey
}

\author{
Eila K Watson (D) , ${ }^{1}$ Jo Brett, ${ }^{1}$ Harriet Hay, ${ }^{2}$ Cara Witwicki, ${ }^{2}$ Anna Perris, ${ }^{2}$ \\ Alan J Poots, ${ }^{2}$ Steve Sizmur, ${ }^{2}$ Zahir Soonawalla, ${ }^{3}$ Amy Tallett $^{2}$
}

To cite: Watson EK, Brett J, Hay $\mathrm{H}$, et al. Experiences and supportive care needs of UK patients with pancreatic cancer: a cross-sectional questionnaire survey. BMJ Open 2019;9:e032681. doi:10.1136/ bmjopen-2019-032681

- Prepublication history for this paper is available online. To view these files, please visit the journal online (http://dx.doi. org/10.1136/bmjopen-2019032681).

Received 01 July 2019 Revised 26 September 2019 Accepted 30 September 2019

Check for updates

(C) Author(s) (or their employer(s)) 2019. Re-use permitted under CC BY-NC. No commercial re-use. See rights and permissions. Published by BMJ.

${ }^{1} 0 x$ ford Institute of Nursing, Midwifery and Allied Health Research, Faculty of Health and Life Sciences, Oxford Brookes University, Oxford, UK

${ }^{2}$ Picker Institute Europe, 0xford, UK

${ }^{3}$ Oxford University Hospitals NHS Foundation Trust, Oxford, UK

Correspondence to Professor Eila K Watson; ewatson@brookes.ac.uk

\section{ABSTRACT}

Objectives Patients diagnosed with pancreatic cancer have the poorest survival prognosis of any cancer. This survey aimed to describe their experiences of care and supportive care needs to inform future service provision. Design Cross-sectional questionnaire survey of patients with pancreatic cancer in the UK.

Setting Individuals at any stage along the care pathway were recruited via five National Health Service sites in the UK, and online, from January to June 2018.

Participants 274 individuals completed the questionnaire (78\% (215) were completed online). Approximately half of participants were diagnosed within the last year (133/274). Of 212 providing gender details, 82 were male and 130 were female. Ninety per cent (192/213) described themselves as White British.

Primary outcome measures Experiences of communication and information; involvement in treatment decisions; supportive care needs.

Results Communication with, and care received from, clinical staff were generally reported positively. However, $29 \%(75 / 260)$ of respondents did not receive enough information at diagnosis, and $10 \%$ (25/253) felt they were not involved in decisions about their treatment, but would have liked to be. Supportive care needs were greatest in psychological and physical/daily living domains. 49\% (108/221) of respondents reported one or more moderate/ high unmet needs within the last month, of which the most commonly reported were: dealing with uncertainty about the future; fears about the cancer spreading; not being able to do things they used to; concerns about those close to them; lack of energy; anxiety; feelings of sadness and feeling down/depressed. Experiences were poorer, and unmet supportive care needs greater, in patients with unresectable disease.

Conclusions Patients with pancreatic cancer have unmet information and support needs across the cancer trajectory. Psychological and physical support appears to be the biggest gap in care. Needs should be assessed and supportive care interventions implemented from the point of diagnosis, and monitored regularly to help patients live as good a quality of life as possible.

\section{INTRODUCTION}

Pancreatic cancer is the 11th most common cancer in the UK, accounting for $3 \%$ of all new cancer cases (2015). ${ }^{1}$ There are around 9800

\section{Strengths and limitations of this study}

- Patients with pancreatic cancer are underrepresented in psychosocial research and this survey provides important insight into their experiences and needs.

- Cognitive testing of the questionnaire was undertaken with a sample of patients, to maximise consistency of responses.

- The study achieved a good sample size, but generalisation is limited by the sampling method used.

- Recruitment through National Health Service clinics was low and most participants were recruited using online methods.

- The sample over-represented people with resectable (better prognosis) disease.

new pancreatic cancer cases in the UK every year (2013-2015). ${ }^{1}$ Around a fifth (21\%) of people diagnosed with pancreatic cancer survive their disease for 1 year or more, and only around $3 \%$ for 5 years or more. ${ }^{1}$ Survival has shown little improvement in the last 40 years, and pancreatic cancer is the fifth most common cause of cancer death in the UK. ${ }^{1}$

Poor prognosis results from a combination of late diagnosis, aggressive tumour biology, complex surgical procedures and a lack of effective systemic treatments. Only around $10 \%-15 \%$ of patients are eligible for tumour resection, and approximately $28 \%$ of patients have chemotherapy as part of their primary cancer treatment. ${ }^{2}$ Newer treatments offer optimism, and can help some patients to live for many months or even years. ${ }^{3}$ For most, however, supportive care is the mainstay of management

Pancreatic cancer is associated with marked symptoms that have a severe impact on quality of life. The early identification and palliation of symptoms is therefore an important aspect of patient care. There is evidence to suggest that the severity of these symptoms can predict prognosis and survival. ${ }^{2}$ Given the nature and prognosis of the disease, it 
is unsurprising that studies have reported diminished quality of life in patients with pancreatic cancer when compared with other cancer populations and the general population. ${ }^{4}$ However, the literature on patient experiences of pancreatic cancer is limited.

Patient experience is increasingly recognised as one of the central components of healthcare quality, and positive associations have been identified between patient experience, patient safety and clinical effectiveness. ${ }^{5}$ Therefore, it is essential to understand people's experiences of care to inform where there is room for improving care delivery and quality. The National Cancer Patient Experience Survey has been conducted annually in England since 2010 to understand and monitor national progress on cancer care. However, the survey is generic to all cancer types, and samples only those with a hospital inpatient stay or day attendance for treatment within a specific 3-month sampling period, with invites sent several months later. ${ }^{6}$ An examination of non-response to this survey found that patients with pancreatic cancer were around five times more likely to have died between sampling and survey mail-out. ${ }^{7}$ As a result, the number of patients with pancreatic cancer responding to the survey is relatively low, and data specific to these patients are not available and are instead grouped alongside other cancer types.

There is a clear need for improved understanding of the physical and emotional well-being of patients with pancreatic cancer, and factors that may help improve their quality of life. This study therefore set out to explore the care experiences and supportive care needs of patients in the UK diagnosed with pancreatic cancer.

\section{METHODS \\ Design}

The study used a cross-sectional survey design. We used the Strengthening the Reporting of Observational Studies in Epidemiology cross-sectional checklist when writing our report. ${ }^{8}$

\section{Survey development}

A draft question set was developed drawing on Picker's existing bank of validated patient experience questions, guided by the Principles of Person-Centred Care, ${ }^{9}$ to enquire about experiences of care at the time of diagnosis, during treatment and beyond.

With permission from the authors, a modified version of the Supportive Care Needs Survey-Short Form (SCNS-SF) ( 34 items) ${ }^{1011}$ was incorporated into the questionnaire. It measures needs across five domains: psychological; physical/daily living; health system/information; patient care/ support; sexuality. Participants were asked to score their need for help in the last month for each item as either no need, need met, low need for help, moderate need for help, or high need for help. The SCNS measure is generic to all cancers, so while we did not remove any items, we included an additional five items specific to pancreatic cancer. These were identified in our literature review and discussions with clinical experts as being particularly relevant to patients and related to:

- Problems with sleeping.

- Nausea and vomiting (eg, feeling/being sick).

- Digestion problems (eg, bowel problems, bloating, wind, discomfort).

- Changes to appetite/body weight.

- Itchiness.

Standard sociodemographic information was also collected.

The cognitive process of responding to the survey questions was also considered, ${ }^{12} 13$ seeking to establish consistency in question comprehension, information retrieval and response. Thirteen cognitive interviews were conducted with patients with a pancreatic cancer diagnosis. Minor amendments to the format and instruction of the SCNS were made following these interviews. Comparability was maintained with the original version, yet the questions were found by participants to be easier to complete once the amendments had been made. In addition, some of the patient experience questions were reworded for clarity; for example, the question, 'How do you feel about the amount of information you were given when first diagnosed', was changed to, 'How do you feel about the amount of information you were given around the time you were diagnosed'. This was in response to feedback that patients struggled to recall the conversation at the exact time of diagnosis.

The final questionnaire addressed the following topic areas: experiences of care (communication and information); supportive care needs (SCNS-SF+five additional items); financial impact of diagnosis; overall quality of life and health rating; and demographic details. The questionnaire also included four open-ended questions where participants could add more detail about their responses (data not presented). A copy of the questionnaire, and the free-text data set, is available on request from the authors.

\section{Patient and public involvement}

Pancreatic Cancer UK provided comments on the draft questionnaire and study design. Thirteen individuals with pancreatic cancer, identified with the help of Pancreatic Cancer UK and the Oxford University Hospitals Foundation Trust pancreatic cancer team, helped to test the questionnaire.

\section{Study population}

Anyone aged 18 and over in the UK with a diagnosis of pancreatic or ampullary cancer was eligible to take part in the survey. A small proportion of patients who are initially suspected to have pancreatic cancer eventually prove to have ampullary cancer. They are treated similarly. ${ }^{14}$ The authors therefore felt that it was preferable to include both diagnoses and acknowledge that a small minority of patients included in the survey may not have pancreatic cancer. 


\section{Sampling}

Recruitment took place between January and June 2018. Five National Health Service (NHS) centres (four in England and one in Scotland) distributed questionnaires to eligible patients attending oncology clinics during the recruitment window. Centres were asked to approach all eligible patients and give them brief information about the survey and, to those who were interested, a study information pack (containing an invitation letter, study information sheet, copy of the study questionnaire and a reply paid envelope). The approach to patients was made by a member of the clinical team, most often the clinical nurse specialist.

In addition, an online version of the questionnaire was made available. This was promoted by Picker, Oxford Brookes University and Pancreatic Cancer UK, using relevant websites and social networks including Twitter, Facebook and Health Unlocked. Events hosted by Pancreatic Cancer UK, and attended by health professionals and patients, were also used to publicise the survey.

\section{Consent}

As approved by the National Research Ethics Service, informed consent was implicit by return of a paper completed questionnaire. This was made clear in the study information. Consent for the online survey was indicated by a respondent submitting their responses or by indicating at the beginning of the survey that their partial responses may be used should they not reach the end.

\section{Statistical analysis}

The completed paper questionnaires were returned to the research team for manual data entry. The online questionnaire was created in Snap (version 11). Snap WebHost was used to collect online responses. At the end of fieldwork, the online data were imported into SPSS (V.25) and combined with paper responses. Frequencies and proportions were used to summarise questionnaire responses. We did not impute values because we were unable to reject the hypothesis of missing not at random in what was already a non-randomised sample. Instead, we used all the data for each individual analysis and provide the denominator used in each case. Where appropriate, results were cross-tabulated by patients with resectable (undergone surgery) and unresectable (had not undergone surgery and/or no surgery planned) disease. Individuals who indicated surgery was planned were removed from these analyses. Statistical tests of inference were not conducted, as the non-random sample precludes generalisation to the population without bias. CIs (95\% CIs) for proportions are provided to give insight into differences within the sample. For analysis of the SCNS-SF34 questions, the original 34 items were used to calculate five domain scores: psychological, physical/daily living, health system/information, patient care/support, and sexuality. Mean normalised domain scores were calculated using the SCNS authors' published procedure. ${ }^{11}$ Briefly, this involved assigning a score of 1 (no need) to 5
Table 1 Participant sociodemographic and clinical characteristics

\begin{tabular}{|c|c|c|}
\hline & $\mathbf{n}$ & $\%$ \\
\hline \multicolumn{3}{|l|}{ Gender } \\
\hline Male & 82 & 38.7 \\
\hline Female & 130 & 61.3 \\
\hline Missing & 62 & \\
\hline \multicolumn{3}{|l|}{ Age group } \\
\hline $31-50$ years & 25 & 11.8 \\
\hline $51-60$ years & 50 & 23.7 \\
\hline $61-70$ years & 82 & 38.9 \\
\hline$>70$ years & 54 & 25.6 \\
\hline Missing & 63 & \\
\hline \multicolumn{3}{|l|}{ Ethnic group } \\
\hline White-British & 192 & 90.1 \\
\hline White-Other & 12 & 5.6 \\
\hline Other & 9 & 4.2 \\
\hline Missing & 61 & \\
\hline \multicolumn{3}{|l|}{ Time since diagnosis } \\
\hline$<6$ months & 82 & 29.9 \\
\hline 6 months to 2 years & 104 & 38.0 \\
\hline$>2$ years & 88 & 32.1 \\
\hline \multicolumn{3}{|l|}{ Treatment } \\
\hline Surgery & 29 & 11.3 \\
\hline Surgery+chemotherapy & 114 & 44.4 \\
\hline Chemotherapy & 85 & 33.1 \\
\hline Surgery and/or chemo planned & 7 & 2.7 \\
\hline No active treatment & 17 & 6.6 \\
\hline Treatment plan unknown & 5 & 1.9 \\
\hline Missing & 17 & \\
\hline
\end{tabular}

(high unmet need) to each SCNS item. The item scores are summed for each domain for each person, and the normalised score calculated, adjusting for the number of questions and maximum response value.

\section{RESULTS}

\section{Study population}

Two hundred and seventy-four completed questionnaires were received (215 online; 59 paper). Sample sociodemographic and clinical characteristics (self-reported) are summarised in table 1 . Some participants did not provide answers to all demographic questions asked, and individual questions were analysed according to the number of respondents to those questions. The mean age of participants was 64 years. Sixty-one per cent (130/212) were female. Forty-nine per cent $(133 / 274)$ of the sample were diagnosed within the last year. Fifty-five per cent $(143 / 258)$ had undergone surgery to remove part of the pancreas; $34 \%(87 / 258)$ were not going to have such 
surgery, $4 \%(11 / 258)$ reported surgery was planned and $7 \%(17 / 258)$ did not know if they would undergo surgery.

\section{Experiences of communication and information}

Most patients reported positive experiences of care. Thirty-eight per cent (103/271) of patients felt their diagnosis had definitely been given in a sensitive way. Participants who had had a pancreatic resection were more likely than unresectable patients to feel that their diagnosis had definitely been given sensitively (39\%; $55 / 142$, $95 \%$ CI $31 \%$ to $47 \%$ vs $31 \% ; 27 / 86,95 \%$ CI $22 \%$ to $42 \%$ ).

Over one-quarter of participants felt they had not been given enough information around the time of diagnosis $(29 \%, 75 / 260)$. A greater proportion of unresectable patients (37\%; $32 / 87,95 \%$ CI $27 \%$ to $42 \%$ ) than those who had had a pancreatic resection $(27 \% ; 38 / 143,95 \%$ CI $20 \%$ to $34 \%$ ) responded in this way.

\section{Involvement in treatment decision-making}

Ten per cent of all participants (25/253) felt they had not been involved in decisions about their treatment and care, and would have liked this, with unresectable patients more likely than resectable patients to report this $(16 \%$; $13 / 83,95 \%$ CI $9 \%$ to $25 \%$ vs $6 \%$; $9 / 140,95 \%$ CI $3 \%$ to $11 \%)$. Less than two-thirds of participants $(60 \% ; 164 / 251)$ responded that staff definitely talked to them about their care and treatment in a way they could understand. Fewer patients with unresectable disease responded in this way ( $52 \% ; 43 / 82,95 \%$ CI $42 \%$ to $63 \%$ vs $72 \% ; 100 / 139,95 \%$ CI $64 \%$ to $79 \%$ ).

\section{Dietary information and therapy}

Most participants in this survey reported receiving pancreatic enzyme replacement therapy (PERT, 83\%; 214/259). A further 5\% (14/259) had been prescribed PERT but were not currently taking it. Thirty-three per cent $(76 / 228)$ of participants prescribed PERT felt they had received insufficient information about it.

\section{Supportive care needs}

The mean normalised domain scores for the SCNS-SF34 items were highest (indicating a greater need) for the psychological and physical/daily living domains (both scoring $>25$ ), and lower in the information, care and sexuality domains (scores <20) (table 2). For the SCNS-SF34 items, $86 \%(190 / 220)$ of respondents reported having at least one supportive care need, and 48\% (105/220) reported one or more moderate or high unmet needs. Using our extended 39-item version of the SCNS, 87\% $(193 / 221)$ of participants reported having one or more unmet supportive care needs and 49\% (108/221) reported one or more moderate or high unmet needs. All SCNS scores were higher in unresectable patients compared with resectable patients. The 10 individual survey items which were most commonly cited as a moderate or high unmet need are presented in table 3 for the overall sample, with the corresponding proportions for resectable and unresectable patients. The level of unmet need was greater in the unresectable group. For unresectable patients digestion problems $(29 \% ; 18 / 62)$ and feelings about death and dying $(27 \% ; 17 / 63)$ were included in the 10 most commonly reported moderate/ high needs, whereas for the resectable group having access to a professional counsellor $(18 \% ; 19 / 105)$ was included (data not shown).

\section{Financial impact}

Just over half of participants (52\%; 111/214) felt the diagnosis of pancreatic cancer had negatively impacted on their day-to-day financial situation. Around a third $(30 \% ; 64 / 212)$ of participants had received financial advice, which they found useful. However, 28\% (59/212) needed financial advice or did not find the advice they had received to be helpful.

\section{DISCUSSION}

This study provides valuable information on the care experiences and supportive care needs of patients living with a diagnosis of pancreatic cancer in the UK. While overall patient experiences of care were positive, the study highlights some important gaps across the patient pathway, including for longer term survivors. Participants with unresectable disease expressed greater unmet needs and poorer experiences overall than patients who had received surgical resection. In the UK, surgical resection rates are around $10 \%-15 \%,{ }^{2}$ and our sample therefore considerably over-represented patients with resectable disease $(56 \%)$ and therefore a better prognosis. The

Table 2 SCNS-SF34 domain scores

\begin{tabular}{|c|c|c|c|c|c|c|c|c|c|}
\hline \multirow[b]{2}{*}{ Domain score } & \multicolumn{3}{|c|}{ Total sample* } & \multicolumn{3}{|c|}{ Resectable patients } & \multicolumn{3}{|c|}{ Non-resectable patients } \\
\hline & $\mathbf{n}$ & Mean & SD & $\mathbf{n}$ & Mean & SD & $\mathbf{n}$ & Mean & SD \\
\hline Psychological & 212 & 31.2 & 30.6 & 120 & 27.8 & 29.3 & 67 & 36.5 & 33.7 \\
\hline Information & 203 & 19.7 & 26.4 & 114 & 15.8 & 23.2 & 67 & 25.3 & 30.6 \\
\hline Care & 201 & 15.9 & 24.9 & 114 & 13.8 & 22.0 & 65 & 20.3 & 30.4 \\
\hline
\end{tabular}

${ }^{*}$ The number of missing responses varied by SCNS-SF34 items.

SCNS-SF34, Supportive Care Needs Survey-Short Form (34 items). 
Table 3 Most common unmet supportive care needs

\begin{tabular}{|c|c|c|c|c|c|c|}
\hline \multirow{2}{*}{$\begin{array}{l}\text { Supportive Care Needs Survey items* } \\
\text { Uncertainty about the future }\end{array}$} & \multicolumn{2}{|c|}{ Total sample } & \multicolumn{2}{|c|}{ Resectable patients $†$} & \multicolumn{2}{|c|}{ Unresectable patientsł } \\
\hline & $31.4 \%$ & $58 / 185$ & $28.6 \%$ & $30 / 105$ & $37.3 \%$ & $22 / 59$ \\
\hline Fears about the cancer spreading & $30.1 \%$ & $56 / 186$ & $29.5 \%$ & $31 / 105$ & $33.9 \%$ & $21 / 62$ \\
\hline $\begin{array}{l}\text { Concerns about the worries of those close to } \\
\text { you }\end{array}$ & $24.7 \%$ & $47 / 190$ & $23.4 \%$ & $26 / 111$ & $30.7 \%$ & $19 / 62$ \\
\hline $\begin{array}{l}\text { Worry that the results of treatment are beyond } \\
\text { your control }\end{array}$ & $21.1 \%$ & $39 / 185$ & $16.0 \%$ & $17 / 106$ & $31.0 \%$ & $18 / 58$ \\
\hline Feelings of sadness & $20.5 \%$ & $39 / 190$ & $17.8 \%$ & 19/107 & $27.9 \%$ & $17 / 61$ \\
\hline $\begin{array}{l}\text { Being informed about things you can do to help } \\
\text { yourself feel better }\end{array}$ & $20.4 \%$ & $39 / 191$ & $17.3 \%$ & $19 / 110$ & $28.6 \%$ & $18 / 63$ \\
\hline
\end{tabular}

*The number of missing responses varied by Supportive Care Needs Survey (SCNS) item.

$\dagger$ Patients who were unsure if they would be having a resection or not were excluded.

$\ddagger$ Patients for whom a resection was planned were not included.

findings, thus, clearly indicate considerable room for improvement in the delivery of patient-centred care to patients diagnosed with pancreatic cancer.

More than one-quarter of participants felt information was lacking around the time of diagnosis, and 1 in 10 participants did not feel involved in decisions about their treatment and care, and would have liked to have been. This finding is supported by a previous UK-based qualitative study, which explored decision-making experiences of patients with pancreatic cancer. Participants reported a sense of being pressured to accept treatment, a sense that in a life-threatening situation there are no 'real options', and reported the confusion that can be caused when clinical opinions differ. ${ }^{15}$ Another study highlighted that the patient's perceived level of involvement in decisionmaking depended on the quality of information, communication and involvement during the care period. ${ }^{16}$

One-third of participants felt they had received insufficient information about PERT. This finding is concerning given that enzyme supplementation, when taken correctly, helps prevent weight loss and improve quality and duration of survival. ${ }^{17}$ It is recommended for the majority of patients. ${ }^{18}$ A previous qualitative study also reported poor understanding of the impact of advanced pancreatic cancer on digestion, a lack of awareness of potential interventions to treat pancreatic exocrine insufficiency and a lack of understanding about the symptoms of pancreatic exocrine insufficiency on the part of the participants. ${ }^{19}$

In relation to supportive care needs, we found needs were greatest in the psychological and physical/daily living domains, concurring with the findings of a recent review of the unmet needs of patients with advanced cancer. ${ }^{20}$ Correspondingly, psychological concerns were prominent in the most commonly reported individual moderate or high unmet need, including: dealing with uncertainty, fear of progression of cancer, concerns about family members and feelings of sadness or depression. Lack of energy and not being able to do the things they used to do were commonly reported physical unmet needs. Interestingly, the five additional pancreatic cancerspecific items we included did not emerge in the 10 most common moderate/high unmet needs for the overall sample, although digestion problems were included in the top 10 for patients with unresectable disease.

Our findings regarding supportive care needs differ in some respects from a previous Australian study, which also used the SCNS-SF34 to explore the unmet needs of patients with pancreatic cancer. ${ }^{21}$ Overall, they found a higher level of supportive care needs and whereas we found the scores to be highest for the psychological domain they found the highest score for the physical/ daily living domain, followed by the psychological, health system/information and patient care domains, which all scored equally. They also found $69 \%$ of respondents reporting at least one moderate to high unmet need. However, the median time from diagnosis of study participants was 3 months, whereas $70 \%$ of our respondents were diagnosed more than 6 months ago, which may explain this difference in findings. Nonetheless, 6 of the top 10 reported moderate to high unmet individual needs items were the same.

The findings from our study suggest that despite the recommended introduction of holistic needs assessment and survivorship care planning to cancer care services, ${ }^{22}$ the psychological aspects of being diagnosed with pancreatic cancer are not being adequately addressed in the UK. Recent guidance on the diagnosis and management of pancreatic cancer ${ }^{18}$ highlights the importance of assessing for and supporting the management of psychological distress. However, many services are stretched and do not have the resources in terms of manpower or skills to address psychological concerns. In some centres, there 
are very limited psychological services available. In other centres, the waiting list may be long and thus problematic for patients with a poor prognosis disease. Referral to psychological services provided in the community (in the UK, via the Improving Access to Psychological Therapies programme $\mathrm{e}^{23}$ ), and signposting to charity counselling and advice services could be helpful for some patients and carers.

There are few psychosocial intervention studies in the literature targeting patients with pancreatic cancer. A small pilot study trialled an interdisciplinary supportive care planning intervention comprising quality of life assessment, case presentation at interdisciplinary care meeting and two nurse-administered educational sessions addressing quality of life concerns. ${ }^{24}$ Patients found the intervention useful but further work is needed to confirm this. A previous study has indicated that a home-based walking programme with regular self-monitoring was feasible, safe and significantly improved fatigue, physical functioning and quality of life compared with usual care, ${ }^{25}$ although again further research in this area is needed. A survey of survivors treated for resectable pancreatic cancer in the USA found that over two-thirds of patients expressed interest in exercise and diet intervention programmes. ${ }^{26}$

Our study has also indicated the level of concern patients have about those close to them, and previous research has indicated the health of caregivers is diminished. ${ }^{27}$ The development of interventions to support caregivers as well as patients is therefore important.

\section{Strengths and weaknesses}

Patients with pancreatic cancer are very underrepresented in psychosocial research. The nature and prognosis of the disease makes this a hard-to-reach patient group, yet one with potentially huge needs. This survey provides responses from 274 individuals in the UK and gives important insight into the experiences and needs of people affected by pancreatic cancer. However, our sample cannot be considered representative of the pancreatic cancer patient population in the UK. Response to recruitment through NHS clinics was low. Online recruitment was much more successful, but introduced greater potential for selection bias. The proportion of resected patients (who have a better prognosis) in our sample is much higher than the national average. Related to this, almost one-third of our sample received their diagnosis more than 2 years ago whereas Public Health England's National Cancer Registration and Analysis Service for the period 2006-2015 indicates a 2-year survival in only $11 \%$ of patients and 3-year survival in $8 \%$ of patients. However, our findings provide novel and valuable data on longer term survivors with pancreatic cancer.

\section{CONCLUSION}

Patients with pancreatic cancer have unmet information, communication and support needs across the cancer trajectory, particularly those with unresectable disease. Needs should be assessed, and supportive care interventions implemented from the point of diagnosis, with regular monitoring thereafter to help patients with pancreatic cancer live as good a quality of life as possible. Psychological support emerged as an important gap in care, and interventions to provide psychological support to patients and their families are required. The use of qualitative research methods and potentially the inclusion of carers as well as patients in future research would further our understanding of patient needs and the types of intervention that would be most useful.

Acknowledgements The authors thank all the patients with pancreatic cancer who took the time to complete the survey. The authors thank all of the NHS staff (Sue Wilner, Roopinder Gillmore, Rose Polcaro, Jennifer Clark, Karen Lloyd Jones, Irene Mulvihill, Aileen Aherne, Elspeth Cowan, Melanie Dadkhah-Taeidy, Dawn Elliot, Kay McCallum, Bridget Taylor), and the Thames Valley and South Midlands Clinical Research Network and Pancreatic Cancer UK for their support with recruitment. The authors thank the authors of the SCNS questionnaire for right to use and modify their work.

Contributors EW, JB, ZS: project inception, management and clinical input. AT: project inception, questionnaire design. HH, CW, AP: project management, questionnaire design, qualitative analysis. AJP and SS conducted statistical analysis and interpreted the findings. All contributed to this manuscript and approved the final draft.

Funding This study was funded by the Pancreatic Cancer UK (grant number 2017), and supported by The Fiorina Fund and Shire.

Competing interests None declared.

Patient consent for publication Not required.

Ethics approval The study received approval from Oxford Brookes University Ethics Committee and the National Research Ethics Service (Ref: 17/SC/0414).

Provenance and peer review Not commissioned; externally peer reviewed.

Data availability statement Data are available in a public, open access repository. Data are available upon reasonable request. All data relevant to the study are included in the article or uploaded as supplementary information.

Open access This is an open access article distributed in accordance with the Creative Commons Attribution Non Commercial (CC BY-NC 4.0) license, which permits others to distribute, remix, adapt, build upon this work non-commercially, and license their derivative works on different terms, provided the original work is properly cited, appropriate credit is given, any changes made indicated, and the use is non-commercial. See: http://creativecommons.org/licenses/by-nc/4.0/.

ORCID iD

Eila K Watson http://orcid.org/0000-0002-3592-1315

\section{REFERENCES}

1 Cancer Research UK. Pancreatic cancer statistics. Available: https:// www.cancerresearchuk.org/health-professional/cancer-statistics/ statistics-by-cancer-type/pancreatic-cancer

2 National Cancer Registration. Analysis Service and Cancer Research UK: "Chemotherapy, Radiotherapy and Tumour Resections in England: 2013-2014" workbook. London: NCRAS, 2017.

3 Neoptolemos JP, Palmer DH, Ghaneh P, et al. Comparison of adjuvant gemcitabine and capecitabine with gemcitabine monotherapy in patients with resected pancreatic cancer (ESPAC-4): a multicentre, open-label, randomised, phase 3 trial. The Lancet 2017;389:1011-24.

4 Sugimoto $\mathrm{H}$, Kawashima $\mathrm{H}$, Ohno $\mathrm{E}$, et al. The prognostic factors and trajectory of HRQOL in patients with pancreatic cancer who received psychiatric intervention. J Gastroenterol Hepatol 2016;31:685-90.

5 Bauer MR, Bright EE, MacDonald JJ, et al. Quality of life in patients with pancreatic cancer and their caregivers: a systematic review. Pancreas 2018;47:368-75.

6 Doyle C, Lennox L, Bell D. A systematic review of evidence on the links between patient experience and clinical safety and effectiveness. BMJ Open 2013;3:e001570. 
7 Abel GA, Saunders CL, Lyratzopoulos G. Post-sampling mortality and non-response patterns in the English cancer patient experience survey: implications for epidemiological studies based on surveys of cancer patients. Cancer Epidemiol 2016;41:34-41.

8 von Elm E, Altman DG, Egger M, et al. The strengthening the reporting of observational studies in epidemiology (STROBE) statement: guidelines for reporting observational studies. J Clin Epidemiol 2008;61:344-9.

9 Picker. Picker principles. [2019-05-14]. Available: https://www.picker. org/about-us/picker-principles-of-person-centred-care/

10 Boyes A, Girgis A, Lecathelinais C. Brief assessment of adult cancer patients' perceived needs: development and validation of the 34item supportive care needs survey (SCNS-SF34). J Eval Clin Pract 2009;15:602-6.

11 McElduff P, Boyes A, Zucca A, et al. The supportive care needs survey: a guide to administration, scoring and analysis. Centre for Health Research \& Psycho-Oncology: Newcastle, 2004.

12 Tourangeau R. Cognitive sciences and survey methods. In: Jabine T, Straf M, Tanur J, eds. Cognitive aspects of survey methodology: building a bridge between disciplines. Washington: National Academy Press, 1984: 73-100.

13 Collins D. Pretesting survey instruments: an overview of cognitive methods. Qual Life Res 2003;12:229-38.

14 Rees JRE, Macefield RC, Blencowe NS, et al. A prospective study of patient reported outcomes in pancreatic and peri-ampullary malignancy. World J Surg 2013;37:2443-53.

15 Ziebland S, Chapple A, Evans J. Barriers to shared decisions in the most serious of cancers: a qualitative study of patients with pancreatic cancer treated in the UK. Health Expect 2015;18:3302-12.

16 Ibrahim F, Sandström P, Björnsson B, et al. 'I want to know why and need to be involved in my own care...': a qualitative interview study with liver, bile duct or pancreatic cancer patients about their experiences with involvement in care. Support Care Cancer 2019;27:2561-7.
17 Roberts KJ, Bannister CA, Schrem H. Enzyme replacement improves survival among patients with pancreatic cancer: results of a population based study. Pancreatology 2019;19:114-21.

18 National Institute for Health and Care Excellence. Pancreatic cancer in adults: diagnosis and management. NICE, London, 2018. Available: https://www.nice.org.uk/guidance/ng85/chapter/ Recommendations\#nutritional-management

19 Gooden HM, White KJ. Pancreatic cancer and supportive care-pancreatic exocrine insufficiency negatively impacts on quality of life. Support Care Cancer 2013;21:1835-41.

20 Wang T, Molassiotis A, Chung BPM, et al. Unmet care needs of advanced cancer patients and their informal caregivers: a systematic review. BMC Palliat Care 2018;17:96.

21 Beesley VL, Janda M, Goldstein D, et al. A tsunami of unmet needs: pancreatic and ampullary cancer patients' supportive care needs and use of community and allied health services. Psychooncology 2016;25:150-7.

22 NHS England. Cancer strategy implementation plan. Available: https://www.england.nhs.uk/cancer/strategy/

23 Hassan S, Bennett K, Serfaty M. Delivering cognitive behavioural therapy to advanced cancer patients: a qualitative exploration into therapists' experiences within a UK psychological service. Clin Psychol Psychother 2018;25:565-74.

24 Sun V, Ruel N, Chung V, et al. Pilot study of an interdisciplinary supportive care planning intervention in pancreatic cancer. Support Care Cancer 2016;24:3417-24.

25 Yeo TP, Burrell SA, Sauter PK, et al. A progressive postresection walking program significantly improves fatigue and health-related quality of life in pancreas and periampullary cancer patients. J Am Coll Surg 2012;214:463-75.

26 Arthur AE, Delk A, Demark-Wahnefried W, et al. Pancreatic cancer survivors' preferences, barriers, and facilitators related to physical activity and diet interventions. J Cancer Surviv 2016;10:981-9.

27 Janda M, Neale RE, Klein K, et al. Anxiety, depression and quality of life in people with pancreatic cancer and their carers. Pancreatology 2017; $17: 321-7$ 